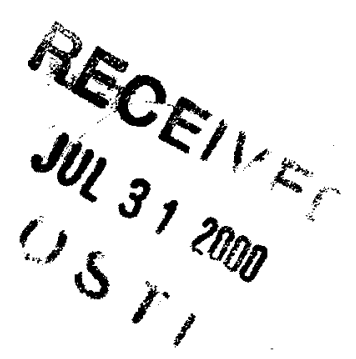

\title{
Simple One-dimensional Transport Code for Magnetized Target Fusion
}

\author{
Stefano Migliuolo \\ Massachusetts Institute of Technology
}

October 30, 1999

\begin{abstract}
A one-dimensional (in space) time-dependent simulation code is developed to study the transport of energy and particles in a field reversed configuration (FRC) plasma that is undergoing radial contraction. This contraction is due to an imploding metallic liner, which is treated through a boundary condition.
\end{abstract}

\section{Introduction}

Magnetized target fusion offers the potential of a low cost "middle-of-theroad" approach to the development of fusion energy [1]-[2]. It involves densities that are intermediate between (and well separated from) magnetic confinement (MFE) and inertial confinement fusion (ICF). It shares with MFE the basic characteristic of thermal insulation by a pre-existing magnetic field and with ICF the presence of a "pusher", in this case a solid metallic liner, to compress the plasma.

MTF may be separated into two efforts: 1) the implosion of the liner, and 2) the confinement and burn of the target fuel. The first has recently met with considerable success; liners have been accelerated to velocities of several $\mathrm{cm} /$ microsecond with good material stability. The second, involving thermal insulation of the plasma under the action of the liner remains to be demonstrated.

It was shown [1] that MTF has (at least) one considerable advantage from the point of view of thermal transport: it may be physically plausible 


\section{DISCLAIMER}

This report was prepared as an account of work sponsored by an agency of the United States Government. Neither the United States Government nor any agency thereof, nor any of their employees, make any warranty, express or implied, or assumes any legal liability or responsibility for the accuracy, completeness, or usefulness of any information, apparatus, product, or process disclosed, or represents that its use would not infringe privately owned rights. Reference herein to any specific commercial product, process, or service by trade name, trademark, manufacturer, or otherwise does not necessarily constitute or imply its endorsement, recommendation, or favoring by the United States Government or any agency thereof. The views and opinions of authors expressed herein do not necessarily state or reflect those of the United States Government or any agency thereof. 


\section{DISCLAIMER}

Portions of this document may be illegible in electronic image products. Images are produced from the best available original document. 
even under the extreme circumstance of Bohm-type energy transport (unlike MFE for which Bohm-level energy losses would make it a "non-starter"). The penalty for such extreme heat losses is that the energy requirement exceeds several MJ per target, as opposed to the far more moderate $30 \mathrm{KJ}$ under "classical" conditions. Of course, one would want to avoid Bohm-like conditions and have more moderate transport.

It is therefore important that the MTF effort have at its disposal a working transport code that may be employed to test and refine transport coefficients, to analyse implosion data, and to forecast optimal regimes of operation. Here, we report on the development of such a code (Version 0.1).

Before describing our work, we note that a one-and-one-quarter transport code was developed in the past [3]-[4] by Werley and co-workers and has been used to simulate [3] FRC plasmas without the effect of an imploding liner. The implosion process, that results in a tenfold radial compression of the plasma is likely to dominate the dynamics of the FRC and this has motivated me to write a completely new code, ab initio, instead of "recoding" the equations of Werley et al.. There are two primary differences between Werley's code and mine:

1. Werley's code uses a coordinate transformation, developed by Caramana [5], that permits following the plasma through its resistive decay. This transformation is similar to an Eulerian $\rightarrow$ Lagrangian transformation, though it is specific to the time evolution of the poloidal magnetic flux. I have opted to go to a fully Lagrangian description in which we follow the plasma throughout the implosion process (the independent "coordinate" is now the differential mass of a fluid element).

2. Werley's code couples the plasma (i.e., the "closed field region") to a vacuum region ("open field") that surrounds the plasma and separates it from the theta-pinch coil. In MTF, the FRC is first produced, then injected (axially) in the target chamber and finally imploded under the action of the liner. I have deliberately chosen to ignore the physics of the liner-vacuum interaction (e.g., is there a current in the liner? Is the flux in the vacuum region conserved during the implosion?,...) and have replaced the open-field region with simple boundary conditions at the plasma edge (defined by "normalized mass $=1$ " at any time interval). In the absence of an open-field region, where the magnetic field lines go to $z= \pm \infty$, it may no longer make sense to include axial motion 
and transport, so my code is truly one-dimensional (radial variation only).

\section{Model equations for the transport simula- tion}

The equations that we solve are continuity (mass conservation), momentum conservation in the radial direction (including inertia), the induction equation (i.e., Ampere's and Faraday's laws plus Ohm's law), and separate equations of energy conservation for electrons and ions. This is analogous to what Werley has done, but for one difference: he replaced the conservation of momentum with a quasi-static radial pressure balance under the assumption that the plasma is evolving slowly compared to, e.g., the travel time of an acoustic wave. This assumption remains to be verified in an implosion scenario and has motivated me to depart from the quasi-static approach.

The equations, and associated boundary conditions are given below in their Lagrangian form. Their common, Eulerian, form are almost exactly the same as those given by Werley (see his Table I), except for the energy equation. We discuss the energy equation in the Appendix.

We briefly recall [6] that in the transformation from an Eulerian to a Lagrangian coordinate system, the convective derivative, $\partial / \partial t+u \partial / \partial r$ (we use a one-dimensional example), collapses to a simple "total" time derivative, $d / d t$. We pay the price in having to compute the radius of a given mass element via an integral relation:

$$
r^{2}(m, t)=r^{2}(0, t)+\int_{0}^{m} \frac{d m}{\pi \rho(m, t)}
$$

which comes trivially from conservation of the differential mass element, in a cylindrical coordinate system: $d m=2 \pi \rho r d r$. In what follows, I will use "total" derivatives, e.g. $d / d m$, to signify differentiation with respect to a Lagrangian coordinate even though most quantities being differentiated are functions of both time and differential mass. Note: the mass, $m$, is the mass per unit length of the cylindrical target plasma. 


\subsection{Continuity}

Here $\rho \approx n_{i} m_{i}$ is the plasma mass density and $U$ its radial velocity:

$$
\frac{d}{d t}\left(\frac{1}{\rho}\right)=\frac{d}{d m}(r U)-\frac{1}{\rho \tau_{p}}
$$

the boundary condition is that the flux $r U$ have zero derivative at the plasma center. Hence $\rho(0, t)$ is a constant in the absence of particle losses, $\tau_{p} \rightarrow \infty$.

\subsection{Momentum}

We write this as:

$$
\frac{d U}{d t}=-r \frac{d}{d m}\left(p+\frac{B_{z}^{2}}{8 \pi}\right)+\dot{U} \delta(r-a)
$$

where the ideal-MHD relation, $J_{\theta}=-(c / 4 \pi)\left(\partial B_{z} / \partial r\right)$ has been used. The boundary condition is imposed at the center, where the gradient of the plasma and magnetic pressures must vanish. As a consequence, $U(0, t)=0$. The last term on the right hand side of this equation is the impulse provided by the imploding liner.

\subsection{Induction}

The equation for the evolution of the magnetic field is given by:

$$
\frac{d B_{z}}{d t}=-B_{z} \rho \frac{d}{d m}(r U)-\rho \frac{d}{d m}\left(\frac{c^{2}}{4 \pi \sigma} r^{2} \rho \frac{d B_{z}}{d m}\right)
$$

One boundary condition, $d B_{Z} / d m=0$ at $m=0$ is already set (see above). The other boundary condition is obtained by requiring that the current vanishes at the plasma edge, $d B_{z} / d m=0$ at $m=m_{p}$ (the total mass of the plasma in the FRC). This replaces the "average beta constraint" used by Werley in his code.

\subsection{Energy}

There are separate equations for the electrons and ions, due to the different heat transport coefficients, as well as the presence of an anomalous term. 
The basic equation, valid for electrons and ions is given by:

$$
\frac{d}{d t}(n T)=-\gamma n T \rho \frac{d}{d m}(r U)+(\gamma-1) Q
$$

where subscripts, e.g. $T_{i}$, have been omitted for brevity and $\gamma$ denotes the ratio of specific heats. The ion conduction term is given by:

$$
Q_{i}=3 \frac{m_{e}}{m_{i}} \frac{n\left(T_{e}-T_{i}\right)}{\tau_{e}}+\frac{f_{i}}{\sigma_{a n}} J_{\theta}^{2}+\rho \frac{d}{d m}\left[r^{2} \rho m_{i} \kappa_{i} \frac{d}{d m}\left(\frac{n T_{i}}{\rho}\right)\right]
$$

the corresponding term for the electrons is:

$$
Q_{e}=-3 \frac{m_{e}}{m_{i}} \frac{n\left(T_{e}-T_{i}\right)}{\tau_{e}}+\left(\frac{1}{\sigma_{c l}}+\frac{1-f_{i}}{\sigma_{a n}}\right) J_{\theta}^{2}+\rho \frac{d}{d m}\left[r^{2} \rho m_{i} \kappa_{e} \frac{d}{d m}\left(\frac{n T_{e}}{\rho}\right)\right]
$$

and, of course, we substitute for $J_{\theta}$ the expression involving the differentiation of the magnetic field, $-(c / 4 \pi)(r \rho)\left(d B_{z} / d m\right)$. The boundary conditions are that the electron/ion pressure gradients must vanish at the center $(m=0)$, while we prescribe the pressures at the edge (i.e., we give a small pedestal value, constant in time).

\section{Code outline and status}

The numerical code is being provided in a separate document. I have tried to be as thorough as possible, commenting it throughout. Basically, we start by inputting physical constants and initial profiles, as a function of mass (equiv. to radius). After the initital $(t=0)$ information is complete and, e.g., the radius is computed for each value of the partial mass, we start advancing the set of equations.

- The continuity and induction equations are solved by a staggered leapfrog method [7] which is second order accurate in time. This is an explicit method and one must be careful to satisfy a Courant condition for the time step relative to the step taken with the mass (or radial) coordinate. Note: in my interim report, I had mentioned using a Two-step Lax-Wendroff scheme. I later decided that there was no substantial benefit, in this case, to offset the increase in bookkeeping (see the note below).

Having obtained the density and velocity at the new time, we proceed to solve: 
- the induction and temperature $\left(T_{i}\right.$ and $\left.T_{e}\right)$ evolution equations. These are solved by a fully implicit Cranck-Nicholson method, [7].

The major points of uncertainty involve the appropriate formulation of the transport coefficients, both anomalous and "classical". It is my impression that Werley used constant coefficients, cf. his Tables II-V. I have chosen to leave $\sigma_{a n}$ as an input constant and employ subroutine calls for the classical coefficients, $\sigma_{c l}$ and $\kappa_{c l}$. Version 0.1 of the code uses Braginskii's expressions [8].

Concerning the methodology, it has become clear to me that one can relatively easily convert this code to a fully implicit version. This would make the Courant condition unnecessary, since the implicit scheme is unconditionally stable. Version 0.2 , if and when I have time to generate it, will be fully implicit. Alternatively, I can suggest a subroutine called VTRANS, written by G. W. Hammett of Princeton Plasma Physics Laboratory, that can be used to do the implicit time-advance of the equations, after some modifications.

Status of the code: I have done some debugging and checking; things appear OK at least at first glance. Please note that though the code appears to be working I have not had the time to check that the numerical answers make physical sense. Things that remain to do:

- Fully debug the code, i.e., verify that the answers for the magnetic field and temperatures at later time steps are correct.

- Vary input parameters to test the stability of the solution

- Figure out the precise Courant condition that needs to be satisfied. Right now I am using the speed of sound and that is very restricitve

- Do a realistic run (many time steps) to really test stability.

\section{Acknowledgment}

This work has been carried out for the Los Alamos National Laboratory, under subcontract no. I04760019-3C (Prime DOE contract W-7405-ENG$36)$. 


\section{Appendix}

The energy equation for the ions/electron in Table I of Werley appears to have a bad misprint. Let me illustrate by considering the standard equations for mass and energy conservation:

$$
\begin{gathered}
\frac{\partial n}{\partial t}+\nabla \cdot(n \vec{v})=A_{n} \\
\frac{\partial T}{\partial t}+(\gamma-1) \nabla \cdot(T \vec{v})=A_{T}
\end{gathered}
$$

where $A_{n}, A_{T}$ are the driving terms that we shall leave unspecified. Do the usual cross-multiplication (by $n$ and $T$ ) of these equations, add them and divide by $\gamma-1$ :

$$
\frac{1}{\gamma-1}\left[\left(\frac{\partial}{\partial t}+\vec{v} \cdot \nabla\right) n T+\gamma n T \nabla \cdot \vec{v}\right]=A_{p}
$$

which can be rewritten as Caramana's [5] equation (4):

$$
\frac{1}{\gamma-1}\left[\frac{\partial}{\partial t}(n T)+\nabla \cdot(n T \vec{v})\right]+n T \nabla \cdot \vec{v}=A_{p}
$$

(just a double-check). Now go back one equation and rewrite the convective piece of the total time derivative plus the compressional term as:

$$
\frac{1}{\gamma-1}(\vec{v} \cdot \nabla) n T+\frac{\gamma}{\gamma-1} n T \nabla \cdot \vec{v}=\frac{\gamma}{\gamma-1} \nabla \cdot(n T \vec{v})-\frac{\gamma-1}{\gamma-1}(\vec{v} \cdot \nabla) n T
$$

Therefore the energy equation ought to read:

$$
\frac{1}{\gamma-1}\left[\frac{\partial n T}{\partial t}+\frac{\gamma}{r} \frac{\partial}{\partial r}\left(r n T v_{r}\right)\right]-v_{r} \frac{\partial n T}{\partial r}+v_{z} \frac{\partial n T}{\partial z}+\frac{\gamma}{\gamma-1} n T \frac{\partial v_{z}}{\partial z}=A_{P}
$$

which has some substantial differences with Werley's equation.

\section{References}

[1] R. E. Siemon et al., Comments Plasma Phys. Cont. Fusion 18, 363 (1999). 
[2] I. R. Lindemuth et al., Bull. Am. Phys. Soc. 43, 1911 (1988).

[3] K. A. Werley, Phys. Fluids 30, 2129 (1987).

[4] M-Y. Hsiao, K. A. Werley, K. M. Ling, Comp. Phys. Comm. 54, 329 (1989).

[5] E. J. Caramana, Phys. Fluids 28, 3557 (1985).

[6] J. J. Duderstadt and G. A. Moses, Inertial Confinement Fusion (Wiley, NY, 1982), Chap. 6.

[7] W. H. Press, et al., Numerical Recipes (Cambridge Univ. Press, 1986), Chap. 17.

[8] S. I. Braginskii, in Reviews of Plasma Pysics, edited by M. Leontovich (Consultants Bureau, NY, 1965), vol. 1, p.205. 